\title{
Relationship Between Public Mental Health and Immune Status During the COVID-19 Pandemic: Cross-Sectional Data from Saudi Arabia
}

This article was published in the following Dove Press journal: Risk Management and Healthcare Policy

\author{
Badrah S Alghamdi $\mathbb{D}^{1,2, *}$ \\ Yasser Alatawi ${ }^{3} *$ \\ Fahad S Alshehri $\mathbb{D}^{4}$ \\ Haythum $O$ Tayeb ${ }^{5}$ \\ Frank I Tarazi ${ }^{6}$ \\ 'Department of Physiology, \\ Neuroscience Unit, Faculty of Medicine, \\ King Abdulaziz University, Jeddah, Saudi \\ Arabia; ${ }^{2}$ Pre-Clinical Research Unit, King \\ Fahad Medical Research Center, King \\ Abdulaziz University, Jeddah, Saudi \\ Arabia; ${ }^{3}$ Department of Pharmacy \\ Practice, Faculty of Pharmacy, University \\ of Tabuk, Tabuk, Saudi Arabia; \\ ${ }^{4}$ Department of Pharmacology and \\ Toxicology, College of Pharmacy, Umm \\ Al-Qura University, Makkah, Saudi \\ Arabia; ${ }^{5}$ Division of Neurology, \\ Department of Internal Medicine, Faculty \\ of Medicine, King Abdulaziz University, \\ Jeddah, Saudi Arabia; ${ }^{6}$ Department of \\ Psychiatry and Neuroscience Program, \\ Harvard Medical School and McLean \\ Hospital, Boston, MA, USA \\ *These authors contributed equally to \\ this work
}

Purpose: Coronavirus disease 2019 (COVID-19) has evolved into a worldwide pandemic and continues to escalate exponentially in many countries across the globe. Recently, higher rates of psychological distress have been reported in several countries during the pandemic. Accordingly, the study aim was to investigate the relationship between public mental health and immune status during the COVID-19 pandemic.

Methods: Participants of this cross-sectional study were 2252 national and foreign residents of Saudi Arabia. We used a web-based self-rated questionnaire to measure the association between psychological distress (Depression, Anxiety and Stress Scales [DASS-21]) and immune status (Immune Status Questionnaire [ISQ]) during the COVID-19 pandemic. We also investigated predictors of reduced immune status using binary logistic regression analyses.

Results: Data from 1721 respondents showed that $17.5 \%$ of participants scored below the immune status cutoff (ISQ $<6$ ). Mean ( \pm standard deviation) depression, anxiety, and stress scores in the reduced immune status group (ISQ $<6$ ) indicated moderate depression, anxiety, and stress $(19.1 \pm 11.4 ; 15.0 \pm 9.6 ; 21.8 \pm 11.2$, respectively) and were significantly higher than scores in the normal immune status group (ISQ $\geq 6)(8.6 \pm 9.1, P<0.0001 ; 5.0 \pm 6.7$, $P<0.0001 ; 9.3 \pm 9.3, P<0.0001$, respectively). The regression analysis showed that age, anxiety, and stress were the only factors that significantly predicted the presence of reduced immune status.

Conclusion: There is an association between mental health problems during the COVID-19 pandemic and immune response in the public, especially in elderly people.

Keywords: mental health, COVID-19, immune status, Immune Status Questionnaire, psychological distress

\section{Introduction}

The concept of a connection between the brain and peripheral organs (a bodymind network), particularly between the endocrine and immune system, can be traced back to antiquity. The new scientific research field of neuroimmunomodulation or psychoneuroimmunology is attracting increasing attention. There is evidence that neuroinflammation plays a crucial role in the pathophysiology of psychiatric disorders, including major depression, and in medical conditions often associated with mental illness, such as cardiovascular and autoimmune diseases, cancer, diabetes mellitus, and arthritis. ${ }^{1}$ Major depression is typically associated with increased rates of morbidity, disability, and suicidal behaviors,
Correspondence: Badrah S Alghamdi Tel +966567764l44

Email basalghamdi@kau.edu.sa
Risk Management and Healthcare Policy 202I:|4 |439-|447

1439 
and imposes a heavy socioeconomic burden on patients, caregivers, and society. For this reason, the World Health Organization has identified major depression as a leading cause of disability worldwide.

Depression is associated with chronic psychosocial stress. ${ }^{2}$ Research has identified a wide range of stressors that disrupt the bidirectional network connection between the brain and immune system, causing neuropsychiatric and immunological disorders. ${ }^{3}$ These stressors can be classified as physical stressors (eg burns, trauma, ischemia, infections, pregnancy, and postpartum) and psychological stressors (eg major life events or environmental events). The effect of stress on the immune system varies according to the nature, intensity, and duration of the stressor. ${ }^{3}$ Moreover, age, sex, psychological status, and genetic factors may affect the immune system response to stress and depression. $^{2}$

Several clinical and experimental studies have reported strong associations between immune dysfunction (both cellular and humoral immunity), stress, and depression. ${ }^{4}$ Chronic stress and depression are associated with increases in inflammatory biomarkers and cortisol levels, leading to changes in serotonergic neurotransmission that may induce mood disorders. ${ }^{5,6}$ One study found that the proinflammatory cytokine interleukin-6 (IL-6) is highly expressed in the blood of depressed patients. ${ }^{7}$ A metaanalysis of 24 studies reported substantially higher levels of IL-6 and tumor-necrosis factor in patients with major depressive disorder. ${ }^{8}$ These data suggest that immune dysfunction and an increase in inflammatory cytokines contribute to the pathophysiology of stress and depressive disorders.

The current coronavirus disease 2019 (COVID-19) pandemic has spread worldwide and has negatively affected mental health. Several recent studies have demonstrated high rates of psychological distress during the pandemic in populations from many countries. A study comparing public health before and during the COVID19 pandemic found that participants reported an increase in negative emotions and a decrease in positive feelings and life satisfaction. ${ }^{9}$ In a Chinese study, $58.3 \%$ of participants reported psychological distress following COVID19-related imposed restrictions on social distancing and shutdowns. ${ }^{10}$ A study of the Iranian population showed higher levels of psychological distress during the COVID19 pandemic and identified several contributing predictors, including work status. ${ }^{11}$ A Brazilian study found moderate mental health distress and identified age and workplace attendance as predictors of distress. ${ }^{12}$ An Italian study also reported higher levels of psychological distress, and identified stressful situations, medical problems, and infected family members as predictors of distress. ${ }^{13}$

Owing to COVID-19 curfews, quarantine, social distancing, shutdowns, and stay-at-home orders, many individuals have experienced profound psychological stress. Such stress may negatively affect the immune system, particularly in individuals experiencing greater psychological distress. There is evidence that patients with a pre-existing mental illness have a higher risk of severe clinical complications from COVID-19 than patients with no previous history of mental illness. ${ }^{14}$ There are no public health studies investigating immune status (IS) and its relation to mental health during the COVID-19 pandemic. In this study, we investigated the effect of COVID-19-induced psychological distress (depression, anxiety, and stress) on IS in a sample of population in Saudi Arabia.

\section{Methods}

\section{Design and Sample}

This study was approved by the research ethics committee of King Abdulaziz University (approval no. 234-20) and conducted in accordance with the Declaration of Helsinki. All participants provided electronic informed consent before participation. We recruited a convenience sample of adult ( $>18$ years old) citizens and residents of Saudi Arabia. Web-based digital data collection is an effective way to obtain insights into the physical and psychological well-being of individuals during pandemics. Therefore, given the travel restrictions and enforcement of social distancing, citizens and residents of Saudi Arabia were recruited via the Internet by distributing a study questionnaire on social media platforms (WhatsApp, Facebook, and Twitter) and institutional email services. Using the institutional account of one of the authors, the questionnaire was generated in Google Forms, a secure online data collection survey tool that allows participants to answer questions conveniently and anonymously. The questionnaire was distributed on May 6, 2020, after 1 month of a nationwide 24-hour curfew. Data collection continued for 1 week, at which point the target sample size was reached.

\section{Inclusion/Exclusion Criteria}

Responses collected from citizens and residents of Saudi Arabia older than 18 years were included in the study. We excluded data from participants who were younger than 18 
years, had a confirmed diagnosis of COVID-19, had direct contact with COVID-19 patients, had a family history of COVID-19 infection, or had inconsistent questionnaire responses.

\section{Measures}

We constructed a simple, self-report questionnaire for this study. The survey was disseminated in both English and Arabic to facilitate the participation of individuals skilled in either language. Subjects were informed about how the collected data would be used and provided informed consent to participate in the study. The survey consisted of three sections. The first section contained questions about sociodemographic variables (age, education, marital status, employment status, income, nationality, and region of residence in Saudi Arabia).

The second section contained questions from the Depression, Anxiety and Stress Scales-21 (DASS-21). ${ }^{15}$ The DASS- 21 is a simple validated tool used to assess psychological distress in both clinical settings and the community. ${ }^{16,17}$ It consists of 21 questions, seven questions for each of the three targeted disorders (depression, anxiety, and stress), Supplementary file 1. Each subscale has cutoff values to categorize symptoms as mild, moderate, severe, or extremely severe. The total DASS-21 score indicates the presence/absence of substantial psychological distress. Previous studies have demonstrated the validity of the DASS-21 in screening for depression, anxiety, and stress with reasonable sensitivity and specificity compared with clinical psychiatric interviews. ${ }^{18}$ The Arabic version of the DASS-21 has been used in previous published studies. ${ }^{19}$ The DASS-21 was recently used in several studies in other countries to assess mental health in the context of the COVID-19 pandemic and has yielded meaningful results. ${ }^{10}$

The third section of the questionnaire contained questions from the Immune Status Questionnaire (ISQ), Supplementary file 2. The ISQ is a simple, validated, and reliable $(r=0.796)$ tool that assesses perceived IS in the public. ${ }^{20}$ It is a shorter form of the Immune Fitness Questionnaire. The ISQ is a self-rated questionnaire and consists of seven symptom items. Participants indicated on a 5-point Likert scale how often they had experienced these symptoms during April 2020 (during the complete curfew period in Saudi Arabia). ${ }^{20}$ The total raw scores were then converted into a final scale score with a cutoff value of 6 (ISQ $<6$ indicates reduced IS). ${ }^{20}$

\section{Statistical Analysis}

We used frequencies, percentages, means, and standard deviations as descriptive statistics. We categorized participants according to their ISQ score as showing reduced IS (ISQ $<6$ ) or normal IS (ISQ $\geq 6$ ). The chi-square test was used to explore the associations between sociodemographic variables and IS groups. We used Student's $t$-test to explore the differences in means between IS groups. Additionally, we performed stepwise binary logistic regression analysis to independently examine sociodemographic and DASS-21 variables as predictors of IS. We calculated the odds ratios (OR) and $95 \%$ confidence intervals (CI) based on the probability of having an ISQ score of less than 6 . We set the threshold for statistical significance at $P<0.05$. We performed statistical analysis using SAS University Edition.

\section{Results}

We collected 2252 survey responses. We excluded 473 responses from healthcare workers to eliminate any chance of direct contact with infected patients. Another 58 responses were excluded because the participants were younger than 18 years or had a confirmed COVID-19 diagnosis, contact with COVID-19 patients, family history of COVID-19 cases, or inconsistent responses. We then analyzed data from the remaining 1721 respondents. Table 1 shows the sociodemographic characteristics of all groups. Most participants were women (68.16\%) and aged 28 years or younger (34.5\%). Only $8.5 \%$ were 59 years or older. Almost $77 \%$ of the sample had a bachelor's degree or higher. Almost $25 \%$ of our participants were unemployed. Most participants (62.81\%) resided in the western region of Saudi Arabia. Only 9.2\% were foreign nationals residing in Saudi Arabia.

Dividing the participants into two groups according to ISQ score showed that $17.5 \%$ of participants had reduced IS (ISQ < 6), with a mean and standard deviation of $3.3 \pm$ 1.8 , and $82.5 \%$ had normal IS (ISQ $\geq 6$ ), with a mean and standard deviation of $9.2 \pm 1.2$. There were statistically significant differences between sociodemographic characteristics and IS groups $(P<0.05)$, except for citizenship and residential region (Table 1).

Figure 1 shows the proportion of different DASS-21 category scores and indicates that approximately one-third of participants experienced psychological distress in the form of depression, anxiety, or stress. IS was associated with severity of psychological distress in a dose-response 
Table I Sociodemographic Characteristics of the Study Sample

\begin{tabular}{|c|c|c|c|c|}
\hline \multirow[t]{2}{*}{ Variable } & \multirow{2}{*}{$\begin{array}{c}\text { Total } \\
\mathbf{N}=|72|\end{array}$} & \multirow{2}{*}{$\begin{array}{l}\text { IS Below Cutoff } \\
\text { Point } \\
\begin{array}{c}\mathbf{N}=30 \text { I (I 17.49) } \\
\mathbf{N}(\%)\end{array}\end{array}$} & \multirow{2}{*}{$\begin{array}{l}\text { IS Above Cutoff } \\
\text { Point } \\
\begin{array}{c}\text { N = I420 (82.5I) } \\
\mathbf{N}(\%)\end{array}\end{array}$} & \multirow[t]{2}{*}{$P$} \\
\hline & & & & \\
\hline \multicolumn{5}{|l|}{ Age (years) } \\
\hline $18-28$ & $593(34.46)$ & $163(54.15)$ & $430(30.28)$ & $<0.0001$ \\
\hline $29-38$ & $414(24.06)$ & 71 (23.59) & $343(24.15)$ & \\
\hline $39-48$ & $321(18.65)$ & $44(14.62)$ & $277(19.51)$ & \\
\hline $49-58$ & $247(14.35)$ & $14(4.65)$ & $233(16.4 I)$ & \\
\hline$\geq 59$ & I 46 (8.48) & $9(2.99)$ & 137 (9.65) & \\
\hline \multicolumn{5}{|l|}{ Sex } \\
\hline Male & $548(31.84)$ & $78(25.91)$ & $470(33.10)$ & 0.0151 \\
\hline Female & $1173(68.16)$ & $223(74.09)$ & $950(66.90)$ & \\
\hline \multicolumn{5}{|l|}{ Educational level } \\
\hline Less than high school & $41(2.38)$ & $3(1.00)$ & $38(2.68)$ & 0.1341 \\
\hline High school & $351(20.40)$ & $72(23.92)$ & $279(19.65)$ & \\
\hline Bachelor's degree & $1092(63.45)$ & $191(63.46)$ & 901 (63.45) & \\
\hline Master's degree & $154(8.95)$ & $25(8.3 \mathrm{I})$ & $129(9.08)$ & \\
\hline Doctoral degree & $83(4.82)$ & $10(3.32)$ & $73(5.14)$ & \\
\hline \multicolumn{5}{|l|}{ Employment } \\
\hline Employed full-time & $627(36.43)$ & $89(29.57)$ & 538 (37.89) & $<0.0001$ \\
\hline Employed part-time & $66(3.83)$ & $13(4.32)$ & $53(3.73)$ & \\
\hline Unemployed & $410(23.82)$ & 75 (24.29) & 335 (23.59) & \\
\hline Student & $359(20.86)$ & $106(35.22)$ & $253(17.82)$ & \\
\hline Retired & $198(11.50)$ & $12(3.99)$ & $186(13.10)$ & \\
\hline Self-employed & $61(3.54)$ & $6(1.99)$ & $55(3.87)$ & \\
\hline \multicolumn{5}{|l|}{ Marital status } \\
\hline Single & $624(36.26)$ & $172(57.14)$ & $452(31.83)$ & $<0.0001$ \\
\hline Married & $1009(58.63)$ & $112(37.21)$ & $897(63.17)$ & \\
\hline Divorced & $68(3.95)$ & $12(3.99)$ & $56(3.94)$ & \\
\hline Widowed & $20(1.16)$ & $5(1.66)$ & $15(1.06)$ & \\
\hline \multicolumn{5}{|l|}{ Monthly income } \\
\hline$<|33|$ USD & 724 (42.07) & $174(56.8 I)$ & $550(38.73)$ & $<0.0001$ \\
\hline |33|-2662 USD & $398(23.13)$ & 65 (21.59) & $333(23.45)$ & \\
\hline 2663-5325 USD & $427(24.8 I)$ & $42(13.95)$ & $385(27.11)$ & \\
\hline$>5325$ USD & $172(9.99)$ & $20(6.64)$ & $152(10.70)$ & \\
\hline \multicolumn{5}{|l|}{ Residential location } \\
\hline Middle region & $270(15.69)$ & $59(19.60)$ & $211(14.86)$ & 0.0570 \\
\hline Western region & $108 \mid(62.8 I)$ & $193(64.12)$ & $888(62.54)$ & \\
\hline Northern region & $94(5.46)$ & II (3.65) & $83(5.85)$ & \\
\hline Southern region & $98(5.69)$ & I (3.65) & $87(6.13)$ & \\
\hline Eastern region & $178(10.34)$ & 27 (8.97) & $151(10.63)$ & \\
\hline \multicolumn{5}{|l|}{ Nationality } \\
\hline Saudi & $1563(90.82)$ & $268(89.04)$ & I 295 (9|.20) & 0.2383 \\
\hline Non-Saudi & $158(9.18)$ & $33(10.96)$ & $125(8.80)$ & \\
\hline
\end{tabular}

Notes: Cutoff point $=6$; N: number of participants.

Abbreviations: USD, United States dollar; IS, immune status. 


\section{Depression}

A

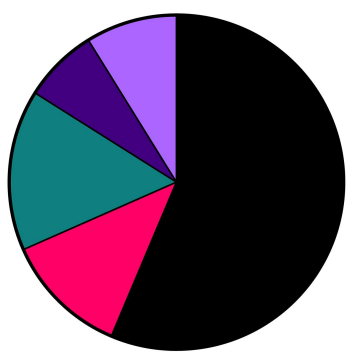

$56.36 \%$ Normal

$12.03 \%$ Mild

$15.57 \%$ Moderate

- $7.21 \%$ Severe

ㄷ.83\% Extremely Severe
B

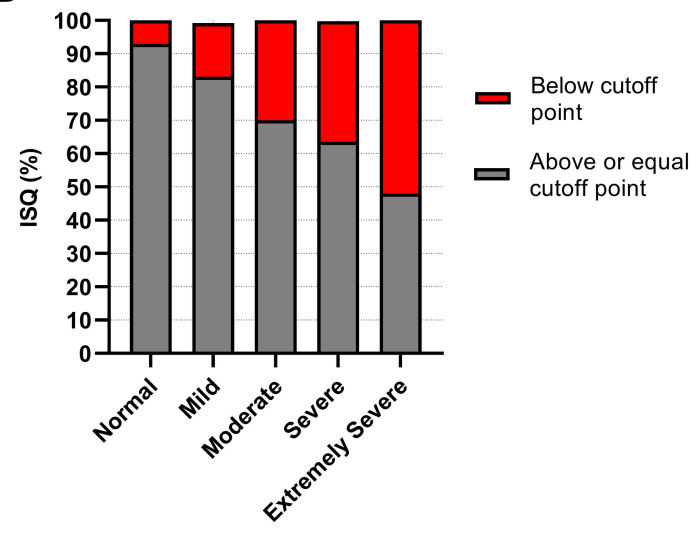

Anxiety

C

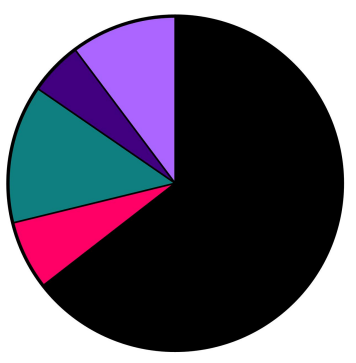

- $64.50 \%$ Norma

$6.68 \%$ Mild

— $13.42 \%$ Moderate

- $5.17 \%$ Severe

ש $10.23 \%$ Extremely Severe

\section{Stress}

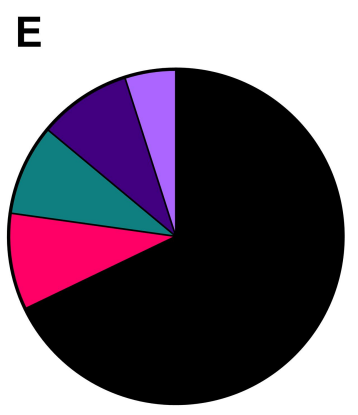

D

$\mathbf{F}$

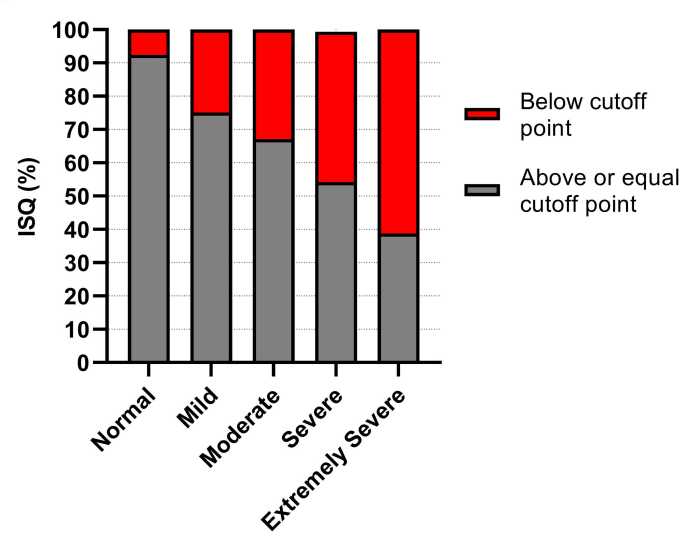

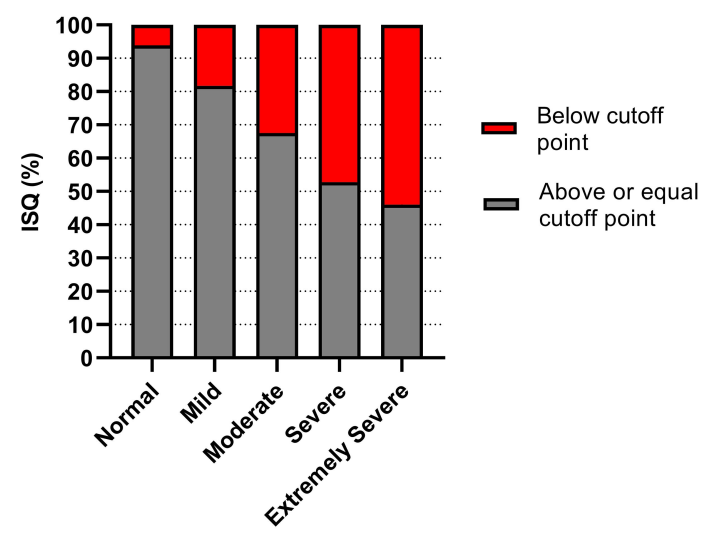

Figure I The proportion of participants in different score categories (normal, mild, moderate, severe, and extremely severe) for each psychological disorder for the whole sample (pie chart) and proportions in each immune status category (below or above cutoff point) for each DASS-2I subscale are shown in the column graph. (A) The proportion of participants in depression score categories. (B) Proportions in each immune status category for depression subscale. (C) The proportion of participants in anxiety score categories. (D) Proportions in each immune status category for anxiety subscale. (E) The proportion of participants in different stress score categories. (F) Proportions in each immune status category for stress subscale.

Abbreviations: DASS-2I, Depression, Anxiety and Stress Scales; ISQ, Immune Status Questionnaire. 
pattern (Figure 1). Figure 2 shows that the mean scores for depression, anxiety, and stress in the reduced IS group were in the range of moderate depression, anxiety, and stress $(19.11 \pm 11.35,14.96 \pm 9.64$, and $21.82 \pm 11.21$, respectively). These DASS-21 scores were significantly higher than those for the normal IS group $(8.6 \pm 9.06$, $P<0.0001 ; 5.04 \pm 6.66, P<0.0001 ; 9.29 \pm 9.28, P<$ 0.0001 , respectively).

Using stepwise binary logistic regression analysis, we investigated the contributions of the different independent sociodemographic variables and DASS-21 subscales of interest to IS (Figure 3). Age, anxiety, and stress were the only factors that significantly predicted the presence of reduced IS (ISQ <6) in the study sample. For instance, participants who reported mild to moderate anxiety were two to four times more likely to have reduced IS than those who did not report anxiety $(\mathrm{OR}=2.3,95 \% \mathrm{CI}=$ $1.3-4.0$ and $\mathrm{OR}=4.3,95 \% \mathrm{CI}=2.8-6.7$, respectively). In addition, participants who reported mild to moderate stress were also more likely to have reduced IS than those who did not report stress $(\mathrm{OR}=1.8,95 \% \mathrm{CI}=1.1-2.8$ and $\mathrm{OR}$ $=2.1,95 \% \mathrm{CI}=1.3-3.4$, respectively).

\section{Discussion}

The COVID-19 pandemic has caused profound psychological and emotional distress in many individuals worldwide. The results from this study show that approximately $33 \%-50 \%$ of subjects reported substantial levels of psychological distress, with approximately 5\%-10\% reporting the most severe symptoms. These data from participants in Saudi Arabia are in line with data collected from other countries during the COVID-19 pandemic. $^{21}$ However, the effects of psychological distress are not restricted to mental health, but can also affect somatic and physical health, as well as non-health-related factors. For example, psychological distress has a substantial negative effect on factors such as pain perception, incidence of arthritis, cardiovascular disease, chronic obstructive pulmonary disease, diabetes, and even mortality rate. ${ }^{22,23}$

Many studies support the hypothesis that there is a bidirectional relationship between the immune system and psychological states, in which psychological distress promotes immune dysfunction and inflammation worsens psychological distress. Several preclinical studies have reported that different types of stressors (eg social isolation, social defeat, unpredictable stress) can induce immune dysfunction in experimental rodents. ${ }^{24-26}$ There is also evidence of an increase in proinflammatory cytokine levels in psychologically distressed patients $^{7,27-31}$ and animal models of depression. ${ }^{32,33}$ These results reflect our own findings, which showed that reduced IS (below the ISQ cutoff point) was positively correlated with the severity of psychological distress, with approximately $50 \%$ of participants in the most severe DASS-21 categories experiencing poor immune response. Moreover, we found that $17.5 \%$ of participants had reduced IS and scored below the immune cutoff point (ISQ < 6) during the COVID-19 pandemic curfew. This is consistent with findings from a previous study indicating anegative correlation between ISQ scores and stress, anxiety, depression, and fatigue. ${ }^{20}$ These findings may explain the higher risk of COVID-19 complications

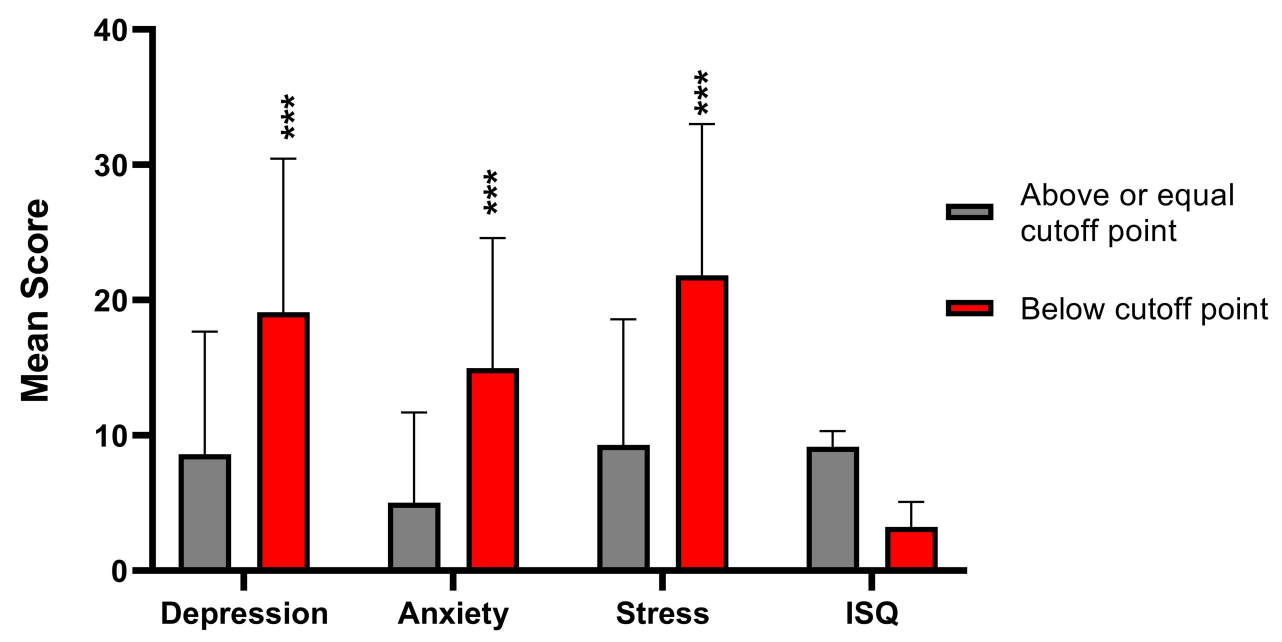

Figure 2 Mean scores for depression, anxiety, stress, and ISQ in two subpopulations. Data are mean \pm standard deviations. Student's $t$-test was used to compare the two subpopulations. $* * * P<0.0001$.

Abbreviation: ISQ, Immune Status Questionnaire. 


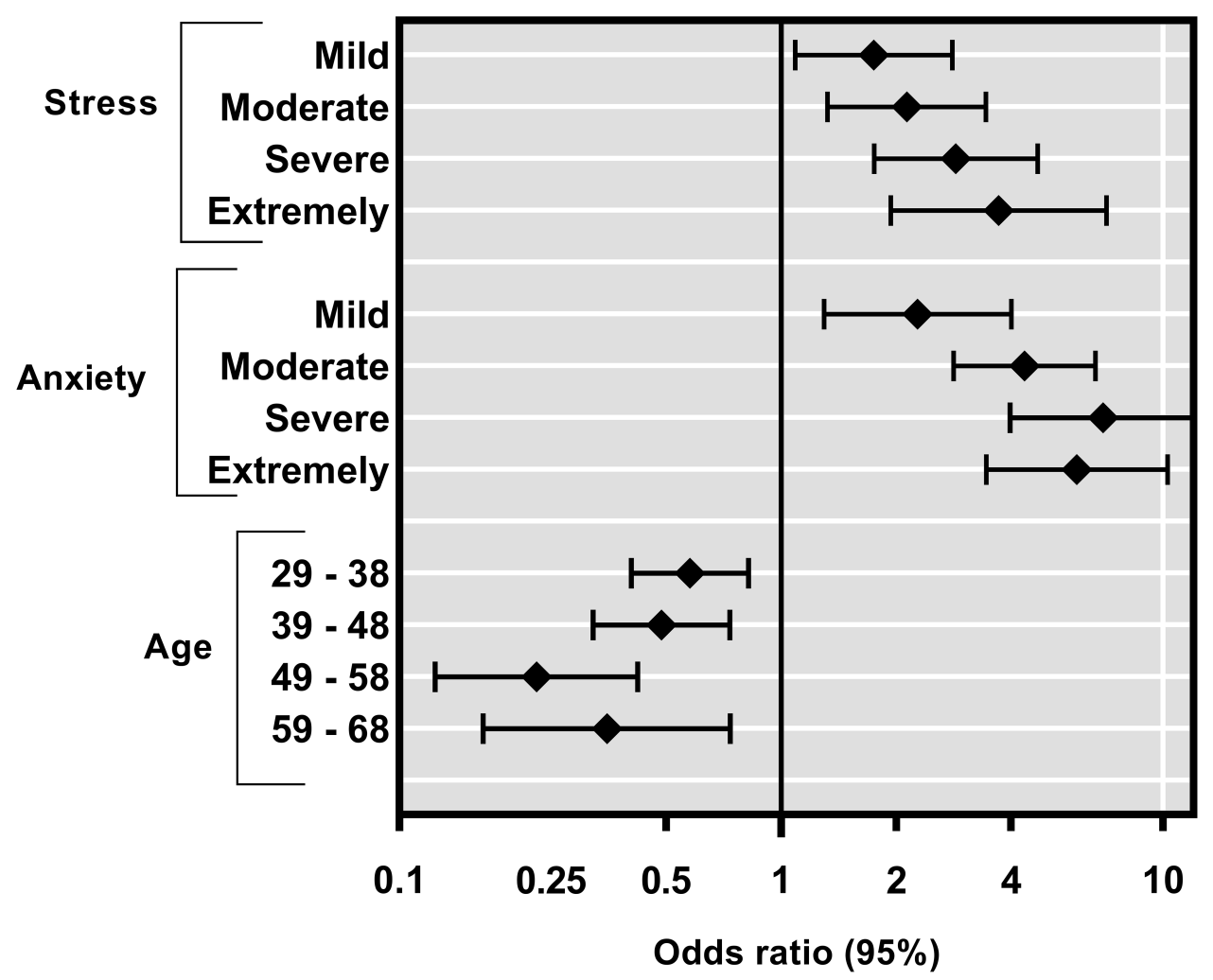

Figure 3 Forest plot of odds ratios and 95\% confidence intervals. The plot represents the correlations between predictors and reduced immune status (ISQ score cutoff point). Each row represents a specific predictor with the corresponding odds ratio (dot) and $95 \%$ confidence intervals (horizontal line).

Abbreviation: ISQ, Immune Status Questionnaire.

in patients with pre-existing mental illness compared with patients with no previous history of mental illness. $^{14}$

The stepwise analysis indicated a potential contribution of independent sociodemographic variables and DASS-21 subscale scores to IS. Age was one potential contributor to poor immune response. This is consistent with previous findings of an age-related decline in immune system function, which increases susceptibility to infections and reduces vaccine efficacy. ${ }^{34}$ Moreover, we showed that anxiety and stress contributed to a decline in IS. This is in line with other studies showing that stress and anxiety can affect the immune system and therefore lead to greater vulnerability to infections and disease. ${ }^{35}$

Previous study findings suggest factors that should be taken into consideration during pandemics to reduce the effect of psychological distress on the immune system. One meta-analysis showed that physical activity can protect from anxiety regardless of demographic factors. ${ }^{36}$ The same study also reported that greater physical activity can confer protection against stress-induced disorders. ${ }^{36}$ Moreover, another meta-analysis reported that yoga may be an effective intervention for individuals with elevated levels of anxiety. ${ }^{37}$ Cognitive behavioral therapy is another effective way to reduce anxiety and psychological distress. ${ }^{38}$ This evidence should guide health policymakers in different countries in generating strategies to overcome or minimize the severe negative effects of national psychological distress during pandemics. A good example is that during the complete curfew, the Saudi government allowed a 1-hour daily walk within a specific radius around the place of residence. There is an urgent need for the implementation of psychological or social interventions in different countries to lessen the negative effects of curfew, quarantine, and social distancing on stress, anxiety, and depression during pandemics.

This study has some limitations. First, owing to the cross-sectional nature of the study, the data cannot be used to infer causality. Second, we used a convenience sampling method. This may have resulted in selection bias if people with very low or very high anxiety levels refrained from participating in the study because they avoided accessing the news media through which the study tool was disseminated. Third, the study was sufficiently 
powered to detect major differences in psychological distress scores but may have not been sufficiently sensitive to detect small differences between specific subgroups (such as older adults, who constituted a relatively small proportion of our sample). Fourth, we did not investigate the medical history of participants and did not exclude any immune-compromised patients or patients with a history of stress-induced disorders. Fifth, we relied on the ISQ survey to predict IS and did not quantify levels of inflammatory biomarkers for each participant.

\section{Conclusion}

To our knowledge, the survey data presented here are the first to demonstrate a relationship between psychological distress and public IS during the COVID-19 pandemic in Saudi Arabia. We showed that the pandemic has resulted in a stressful psychological burden that may negatively affect the IS of the public. This study highlights the harmful effects of pandemic-induced psychological distress on the public. It also identifies an urgent need to implement psychological or social interventions to reduce the negative psychosocial effect of the pandemic on public mental health and to reverse the observed immune system dysfunction.

\section{Author Contributions}

All authors made a significant contribution to the work reported, whether that is in the conception, study design, execution, acquisition of data, analysis and interpretation, or in all these areas; took part in drafting, revising, or critically reviewing the article; gave final approval of the version to be published; have agreed on the journal to which the article has been submitted; and agree to be accountable for all aspects of the work.

\section{Funding}

This research did not receive any specific grant from funding agencies in the public, commercial, or not-forprofit sectors.

\section{Disclosure}

The authors report no conflicts of interest for this work.

\section{References}

1. Murray CJ, Lopez AD. Global mortality, disability, and the contribution of risk factors: Global Burden of Disease Study. Lancet. 1997;349 (9063):1436-1442. doi:10.1016/S0140-6736(96)07495-8

2. Leonard B. Stress, depression and the activation of the immune system. World J Biol Psychiatry. 2000;1(1):17-25. doi:10.3109/ 15622970009150562
3. Reiche E, Morimoto H, Nunes S. Stress and depression-induced immune dysfunction: implications for the development and progression of cancer. Int Rev Psychiatry. 2006;17(6):515-527. doi:10.1080/ 02646830500382102

4. Raison CL, Capuron L, Miller AH. Cytokines sing the blues: inflammation and the pathogenesis of depression. Trends Immunol. 2006;27 (1):24-31. doi:10.1016/j.it.2005.11.006

5. Maddock C, Pariante CM. How does stress affect you? An overview of stress, immunity, depression and disease. Epidemiol Psichiatr Soc. 2001;10(3):153-162. doi:10.1017/S1121189X00005285

6. Tafet GE, Toister-Achituv M, Shinitzky M. Enhancement of serotonin uptake by cortisol: a possible link between stress and depression. Cogn Affect Behav Neurosci. 2001;1(1):96-104. doi:10.3758/ CABN.1.1.96

7. Dantzer R, O'Connor JC, Freund GG, Johnson RW, Kelley KW. From inflammation to sickness and depression: when the immune system subjugates the brain. Nat Rev Neurosci. 2008;9(1):46-56. doi:10.1038/nrn2297

8. Dowlati Y, Herrmann N, Swardfager W, et al. A meta-analysis of cytokines in major depression. Biol Psychiatry. 2010;67(5):446-457. doi:10.1016/j.biopsych.2009.09.033

9. Li S, Wang Y, Xue J, Zhao N, Zhu T. The impact of COVID-19 epidemic declaration on psychological consequences: a study on active Weibo users. Int J Environ Res Public Health. 2020;17 (6):2032. doi:10.3390/ijerph17062032

10. Wang C, Pan R, Wan X, et al. A longitudinal study on the mental health of general population during the COVID-19 epidemic in China. Brain Behav Immun. 2020;87:40-48. doi:10.1016/j. bbi.2020.04.028

11. Jahanshahi AA, Dinani MM, Madavani AN, Li J, Zhang SX. The distress of Iranian adults during the Covid-19 pandemic - More distressed than the Chinese and with different predictors. Brain Behav Immun. 2020;87:124-125. doi:10.1016/j.bbi.2020.04.081

12. Zhang S, Wang Y, Afshar Jahanshahi A, Jia J, Schmitt V. First study on mental distress in Brazil during the COVID-19 crisis. Preprint. 2020.

13. Mazza C, Ricci E, Biondi S, et al. A nationwide survey of psychological distress among Italian people during the COVID-19 pandemic: immediate psychological responses and associated factors. Int J Environ Res Public Health. 2020;17(9):3165. doi:10.3390/ ijerph17093165

14. Lee SW, Yang JM, Moon SY, et al. Association between mental illness and COVID-19 susceptibility and clinical outcomes in South Korea: a nationwide cohort study. Lancet Psychiatry. 2020;7 (12):1025-1031. doi:10.1016/S2215-0366(20)30421-1

15. Henry JD, Crawford JR. The short-form version of the Depression Anxiety Stress Scales (DASS-21): construct validity and normative data in a large non-clinical sample. Br J Clin Psychol. 2005;44 (2):227-239. doi:10.1348/014466505X29657

16. Lovibond PF. Long-term stability of depression, anxiety, and stress syndromes. J Abnorm Psychol. 1998;107(3):520-526. doi:10.1037/ 0021-843X.107.3.520

17. Norton PJ. Depression Anxiety and Stress Scales (DASS-21): psychometric analysis across four racial groups. Anxiety Stress Coping. 2007;20(3):253-265. doi:10.1080/10615800701309279

18. Tran TD, Tran T, Fisher J. Validation of the depression anxiety stress scales (DASS) 21 as a screening instrument for depression and anxiety in a rural community-based cohort of northern Vietnamese women. BMC Psychiatry. 2013;13(1):24. doi:10.1186/1471-244X13-24

19. Alzahrani A, Alghamdi A, Alqarni T, Alshareef R, Alzahrani A. Prevalence and predictors of depression, anxiety, and stress symptoms among patients with type II diabetes attending primary healthcare centers in the western region of Saudi Arabia: a cross-sectional study. Int J Ment Health Syst. 2019;13(1):48. doi:10.1186/s13033019-0307-6 
20. Wilod Versprille LJF, van de Loo AJAE, Mackus M, et al. Development and validation of the Immune Status Questionnaire (ISQ). Int $J$ Environ Res Public Health. 2019;16(23):4743. doi:10.3390/ijerph16234743

21. Alghamdi BS, AlAtawi Y, AlShehri FS, et al. Psychological distress during COVID-19 curfews and social distancing in Saudi Arabia: a cross-sectional study. Res Sq. 2020. doi:10.21203/rs.3.rs-40296/v1

22. Lingard EA, Riddle DL. Impact of psychological distress on pain and function following knee arthroplasty. JBJS. 2007;89(6):1161-1169. doi:10.2106/00004623-200706000-00002

23. de Burgos-lunar C, Gómez-Campelo P, Cárdenas-Valladolid J, et al. Effect of depression on mortality and cardiovascular morbidity in type 2 diabetes mellitus after 3 years follow up. The DIADEMA study protocol. BMC Psychiatry. 2012;12(1):95. doi:10.1186/1471244X-12-95

24. Grippo AJ, Sullivan NR, Damjanoska KJ, et al. Chronic mild stress induces behavioral and physiological changes, and may alter serotonin 1A receptor function, in male and cycling female rats. Psychopharmacology. 2005;179(4):769-780. doi:10.1007/s00213004-2103-4

25. Audet MC, Jacobson-Pick S, Wann BP, Anisman H. Social defeat promotes specific cytokine variations within the prefrontal cortex upon subsequent aggressive or endotoxin challenges. Brain Behav Immun. 2011;25(6):1197-1205. doi:10.1016/j.bbi.2011.03.010

26. Möller M, Du Preez JL, Viljoen FP, Berk M, Emsley R, Harvey BH. Social isolation rearing induces mitochondrial, immunological, neurochemical and behavioural deficits in rats, and is reversed by clozapine or N-acetyl cysteine. Brain Behav Immun. 2013;30:156-167. doi:10.1016/j.bbi.2012.12.011

27. Tuglu C, Kara SH, Caliyurt O, Vardar E, Abay E. Increased serum tumor necrosis factor-alpha levels and treatment response in major depressive disorder. Psychopharmacology. 2003;170(4):429-433. doi:10.1007/s00213-003-1566-z

28. Basterzi A, Aydemir C, Kisa C, et al. IL-6 levels decrease with SSRI treatment in patients with major depression. Hum Psychopharmacol. 2005;20(7):473-476. doi:10.1002/hup.717
29. Tsao CW, Lin YS, Chen CC, Bai CH, Wu SR. Cytokines and serotonin transporter in patients with major depression. Prog Neuropsychopharmacol Biol Psychiatry. 2006;30(5):899-905. doi:10.1016/j.pnpbp.2006.01.029

30. Miller AH, Maletic V, Raison CL. Inflammation and its discontents: the role of cytokines in the pathophysiology of major depression. Biol Psychiatry. 2009;65(9):732-741. doi:10.1016/j.biopsych.2008.11.029

31. Steptoe A, Willemsen G, Owen N, Flower L, Mohamed-Ali V. Acute mental stress elicits delayed increase in circulating inflammatory cytokine levels. Clin Sci. 2001;101:185-192.

32. Elgarf AS, Aboul-Fotouh S, Abd-Alkhalek HA, et al. Lipopolysaccharide repeated challenge followed by chronic mild stress protocol introduces a combined model of depression in rats: reversibility by imipramine and pentoxifylline. Pharmacol Biochem Behav. 2014;126:152-162. doi:10.1016/j.pbb.2014.09.014

33. Lu Y, Ho CS, Liu X, et al. Chronic administration of fluoxetine and pro-inflammatory cytokine change in a rat model of depression. PLoS One. 2017;12(10):e0186700. doi:10.1371/journal.pone.0186700

34. Fuentes E, Fuentes M, Alarcón M, Palomo I. Immune system dysfunction in the elderly. An Acad Bras Cienc. 2017;89(1):285-299. doi:10.1590/0001-3765201720160487

35. Segerstrom SC, Miller GE. Psychological stress and the human immune system: a meta-analytic study of 30 years of inquiry. Psychol Bull. 2004;130(4):601-630. doi:10.1037/00332909.130.4.601

36. Schuch FB, Stubbs B, Meyer J, et al. Physical activity protects from incident anxiety: a meta-analysis of prospective cohort studies. Depress Anxiety. 2019;36(9):846-858. doi:10.1002/da.22915

37. Cramer H, Lauche R, Anheyer D, et al. Yoga for anxiety: a systematic review and meta-analysis of randomized controlled trials. Depress Anxiety. 2018;35(9):830-843. doi:10.1002/da.22762

38. Carpenter JK, Andrews LA, Witcraft SM, Powers MB, Smits JAJ, Hofmann SG. Cognitive behavioral therapy for anxiety and related disorders: a meta-analysis of randomized placebo-controlled trials. Depress Anxiety. 2018;35(6):502-514. doi:10.1002/da.22728
Risk Management and Healthcare Policy

\section{Publish your work in this journal}

Risk Management and Healthcare Policy is an international, peerreviewed, open access journal focusing on all aspects of public health, policy, and preventative measures to promote good health and improve morbidity and mortality in the population. The journal welcomes submitted papers covering original research, basic science, clinical \& epidemiological studies, reviews and evaluations, guidelines, expert opinion and commentary, case reports and extended reports. The manuscript management system is completely online and includes a very quick and fair peer-review system, which is all easy to use. Visit http://www.dovepress.com/testimonials.php to read real quotes from published authors. 